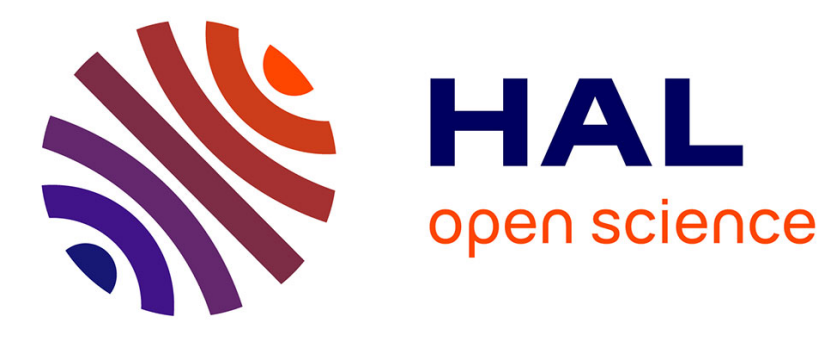

\title{
Impulsion microonde gigawatt en bande $\mathrm{X}$
}

J.M. Buzzi, H.J. Doucet, H. Lamain, C. Rouillé, J. Delvaux, J.C. Jouys

\section{To cite this version:}

J.M. Buzzi, H.J. Doucet, H. Lamain, C. Rouillé, J. Delvaux, et al.. Impulsion microonde gigawatt en bande X. Journal de Physique Lettres, 1978, 39 (2), pp.15-18. 10.1051/jphyslet:0197800390201500 . jpa-00231432

\section{HAL Id: jpa-00231432 https://hal.science/jpa-00231432}

Submitted on 1 Jan 1978

HAL is a multi-disciplinary open access archive for the deposit and dissemination of scientific research documents, whether they are published or not. The documents may come from teaching and research institutions in France or abroad, or from public or private research centers.
L'archive ouverte pluridisciplinaire HAL, est destinée au dépôt et à la diffusion de documents scientifiques de niveau recherche, publiés ou non, émanant des établissements d'enseignement et de recherche français ou étrangers, des laboratoires publics ou privés. 


\author{
Classification \\ Physics Abstracts \\ $41.80 \mathrm{D}-42.52-52.40 \mathrm{~F}-52.60$
}

\title{
IMPULSION MICROONDE GIGAWATT EN BANDE $X$
}

\author{
J. M. BUZZI, H. J. DOUCET, H. LAMAIN, C. ROUILlÉ \\ Laboratoire de Physique des Milieux Ionisés $\left({ }^{*}\right)$, Ecole Polytechnique, 91128 Palaiseau Cedex, France
}

J. DELVAUX et J. C. JOUYS

Commissariat à l'Energie Atomique, Centre d'Etudes de Valduc, France

(Reçu le 5 juillet 1977, révisé le 5 septembre 1977, accepté le 28 novembre 1977)

\begin{abstract}
Résumé. - Une émission Gigawatt en bande $X$ est observée en introduisant un faisceau d'électrons de grande intensité $(v / \gamma \gg 1)$ dans un guide d'onde rempli d'air à basse pression.
\end{abstract}

Abstract. - A Gigawatt pulse of microwaves in the $\mathbf{X}$ band is produced on injecting an intense relativistic electron beam $(v / \gamma \gg 1)$ in a waveguide filled with air at low pressure.

1. Introduction. - L'utilisation des machines à faisceaux d'électrons relativistes a permis d'augmenter de deux à trois décades la puissance des impulsions électromagnétiques en ondes centimétriques et décimétriques. De nombreux dispositifs ont été utilisés, basés soit sur des systèmes à cavités résonnantes issus des dispositifs classiques $[1,2,3]$ soit sur une émission maser mettant en jeu une instabilité faisceau-guide d'onde $[4,5,6]$.

Jusqu'à ce jour tous les dispositifs utilisés fonctionnaient avec des résonances fixées par des paramètres extérieurs au faisceau : champ magnétique imposé, dimension d'une cavité résonnante ce qui entraînait une limitation importante de la puissance. En effet, à forte densité de courant, le champ magnétique du faisceau ou la densité de charge modifient considérablement ces paramètres. Ces effets conduisent à des dispositifs où la valeur des courants utilisés reste en général bien inférieure à la limite du courant d'Alfven $I_{\mathrm{A}}$ [7].

Dans le présent travail nous avons observé une émission microonde en bande $X$ de très grande puissance dans un dipositif où la valeur du courant était bien supérieure à la limite d'Alfven, donc dans des conditions où les champs propres du faisceau loin d'être négligeables peuvent au contraire déterminer de nouveaux mécanismes d'émission.

2. Description du montage expérimental. - Le faisceau d'électrons est produit par une machine

(*) Groupe de recherche du C.N.R.S. constituée d'un générateur de Marx chargeant une ligne $2 \Omega$ et délivrant sur une cathode de graphite de diamètre $4 \mathrm{~cm}$, un courant maximum de $220 \mathrm{kA}$ sous une tension de $350 \mathrm{kV}$. Le temps de montée de l'impulsion était de l'ordre de $50 \mathrm{~ns}$ et la durée de l'impulsion à mi-hauteur voisine de 150 ns.

Cette cathode était placée à $8 \mathrm{~mm}$ d'une anode, feuille de mylar de $6 \mu$ d'épaisseur, aluminisée sur les deux faces, dans une enceinte avec une pression résiduelle $\leqslant 10^{-4}$ torr.

Le faisceau d'électrons était injecté à travers cette feuille dans une enceinte en acier inoxydable comportant deux tubes cylindriques conducteurs reliés par un cône (voir Fig. 1). Le premier tube conducteur était en cuivre feuilleté pour éviter les courants de

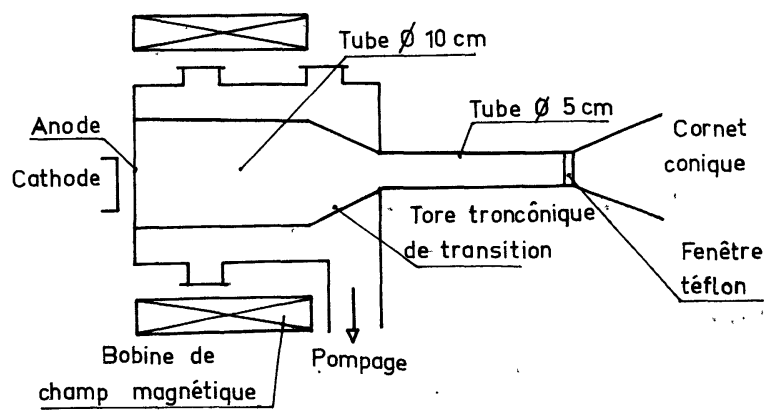

FIG. 1. - Dispositif d'émission Gigawatt de microonde en bande X par injection d'un faisceau intense d'électrons relativistes dans un guide d'onde partiellement 'rempli de gaz.

[Diagram of the device used for Gigawatt $\mathrm{X}$ band emission. An intense relativistic electron beam is introduced in a partially gas filled waveguide.] 
Foucault. Ce tube avait un diamètre de $10 \mathrm{~cm}$ et une longueur de $40 \mathrm{~cm}$. Il était placé derrière l'anode, son axe de révolution coïncidant avec celui de la diode.

La pression dans ce tube pouvait être réglée de $5 \times 10^{-3}$ torr à quelques torrs. Le gaz utilisé était le gaz résiduel à basse pression, et de l'air à pression plus élevée.

Ce premier tube était prolongé par un tube coaxial de diamètre $5 \mathrm{~cm}$ et de longueur $80 \mathrm{~cm}$, en acier inoxydable, centré sur le premier tube à l'aide d'un tore de forme tronconique assurant une transition micro-onde sur une longueur de $10 \mathrm{~cm}$.

L'extrémité du tube cylindrique de $\varnothing 5 \mathrm{~cm}$ était obturée par un hublot en téflon d'épaisseur $5 \mathrm{~mm}$, puis reliée à un cornet conique de grande dimension assurant une bonne adaptation du guide cylindrique de $5 \mathrm{~cm}$ à l'espace libre.

La détection était assurée par un petit cornet mobile, des atténuateurs convenables et une ligne à retard en guides rectangulaires en bande $\mathrm{X}$ permettant l'analyse spectrale du signal. Ce système de détection est schématisé sur la figure 2 .

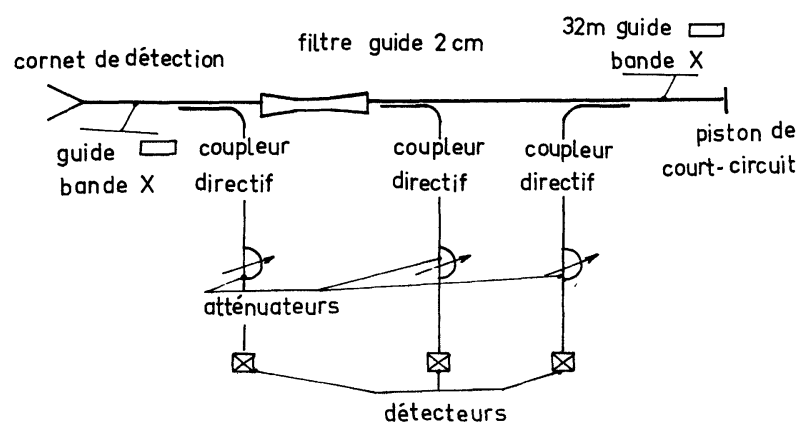

Fig. 2. - Dispositif de mesure de la fréquence et de la puissance de l'onde émise.

[Frequency and power measurement devices.]

Enfin un champ magnétique axial pouvait être ajouté au dispositif par un enroulement à spires jointives placé autour de la chambre d'interaction sur une longueur voisine de celle du tube de $\varnothing 10 \mathrm{~cm}$. Le champ magnétique maximum au centre de ce tube était de l'ordre de 1 Tesla.

3. Résultats expérimentaux. - 3.1 Lorsque le faisceau est introduit dans de l'air à basse pression $5 \times 10^{-3}$ torr à 1 torr, une émission intense est obtenue pendant $30 \mathrm{~ns}$ environ, durée voisine de celle du flash $\gamma$ mesuré au niveau de la diode, durée indicative de la partie grande puissance du faisceau d'électrons.

La forme précise de l'impulsion n'était pas reproductible dans ses détails mais avait en général l'allure présentée sur la figure 3 : une impulsion relativement longue (30-50 ns) de puissance maximum voisine de $500 \mathrm{MW}$ à $1 \mathrm{GW}$. Puis une ou plusieurs impulsions

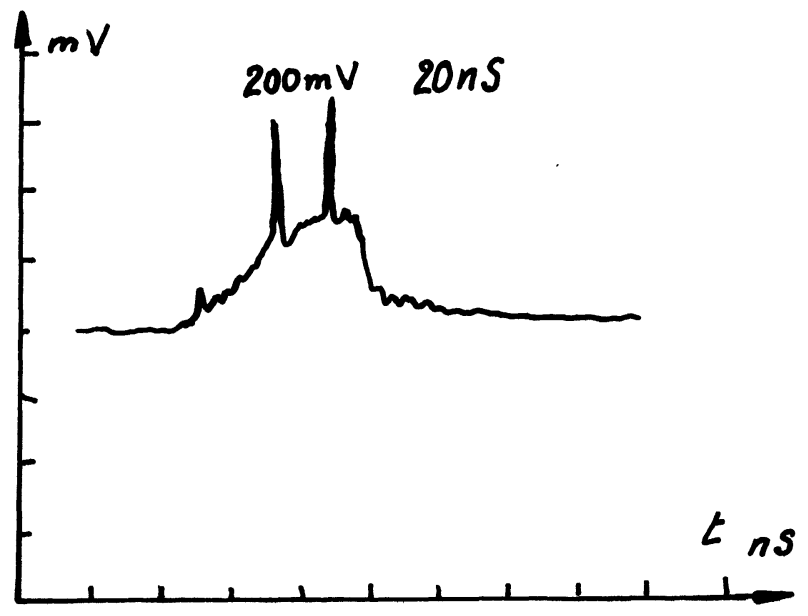

Fig. 3. - Exemple de mesure en fonction du temps de l'émission gigawatt observée.

[Sample of observed Gigawatt emission as a function of time.]

très courtes (quelques ns à $10 \mathrm{~ns}$ ) de puissance estimée à 1 à $2 \mathrm{GW}$. Les puissances ont été mesurées par un étalonnage de cristaux détecteurs IN23B et BAW93 après un soigneux étalonnage des atténuateurs et coupleurs directifs pour les fréquences mesurées et discutées ci-après. Cette mesure de la puissance a été vérifiée en injectant une onde témoin de puissance $100 \mathrm{~kW}$, émise par un magnétron alimenté en impulsion. Cette impulsion témoin était injectée dans le système par un coupleur $3 \mathrm{db}$ assurant l'adaptation entre un guide rectangulaire relié au magnétron et un guide circulaire de diamètre $5 \mathrm{~cm}$ placé entre la fenêtre en téflon et le cornet cylindrique.

3.2 FRÉQUeNCE DE L'ÉMISSION. - La fréquence de l'émission est donnée par le retard du signal dans une ligne dispersive constituée de $63 \mathrm{~m}$ de guide rectangulaire.

On observe pour un même pic d'émission, 3 pics étroits au bout de ligne à retard, ce qui conduit à des signaux de fréquences étroites centrées autour de 9,11 et $14 \mathrm{GHz}$ :

- Ces trois fréquences sont remarquablement reproductibles.

- Ces fréquences sont indépendantes de la valeur de la pression du gaz.

- Ces fréquences sont indépendantes de la valeur du champ magnétique dans l'enceinte d'interaction quand il existe.

- Ces fréquences restent inchangées si le tube de $\varnothing 5 \mathrm{~cm}$ est prolongé jusqu'à quelques $\mathrm{cm}$ de l'anode à l'intérieur du tube de $\varnothing 10 \mathrm{~cm}$.

3.3 Puissance De L'Émission EN FONCTION DE LA PRESSION. - La figure 4 montre les variations de la puissance émise en fonction de la pression, ainsi que l'énergie transportée par le faisceau et mesurée par calorimétrie au bout du tube de $\varnothing 5 \mathrm{~cm}$. 


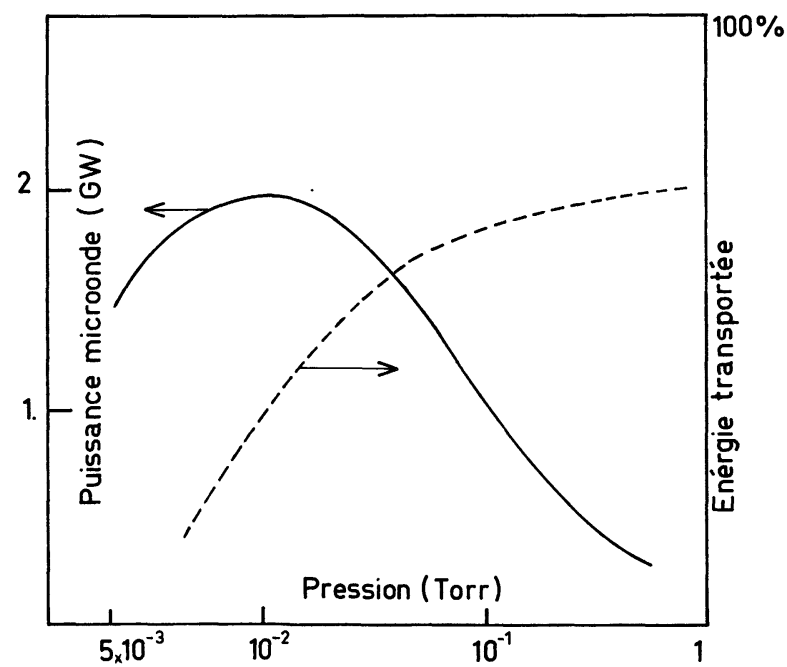

Fig. 4. - Variations de la puissance en fonction de la pression et énergie transportée par le faisceau.

[Emitted power and beam energy at the end of the device as a function of the gas pressure.]

4. Essais d'interprétation. - La valeur du courant utilisée rend inutilisables les mécanismes classiques d'émission [8]. Par contre la charge d'espace du faisceau et son champ magnétique propre peuvent introduire de nouveaux mécanismes que nous discuterons ici très brièvement. Enfin d'autres mécanismes peuvent être envisagés en raison de la production rapide d'un plasma de forte densité surtout à forte pression du gaz [8]. Une étude plus complète étant indispensable, nous nous contenterons ici de souligner les caractéristiques de l'émission qui peuvent faire songer à un mécanisme particulier :

4.1 Effet DE ChARGe D'EsPaCE. - Compte tenu du courant très fort utilisé, une partie des électrons n'est pas transportée, comme le montre les mesures d'énergie transmise, à cause de la charge d'espace des électrons du faisceau. De nombreux électrons sont réfléchis et oscillent autour du plasma anodique comme cela se produit dans la diode reflex génératrice d'importants courants d'ions [9]. Le mouvement non isochrone des électrons dans le puits de potentiel de l'anode peut conduire à une émission cohérente, le regroupement des électrons étant produit par la dépendance de la fréquence d'oscillation des électrons à leur énergie [9]. C'est le mécanisme envisagé pour expliquer l'émission de quelque $100 \mathrm{MW}$ observée dans le vide au N.R.L. à la suite du présent travail.

En faveur de cette théorie, on peut noter que la fréquence attendue aurait le bon ordre de grandeur, et serait indépendante de la pression et du champ magnétique, que le rendement optimum dans le cas du N.R.L. était du même ordre que dans le nôtre.

Par contre on s'attendrait dans ce cas à l'émission d'une seule fréquence avec la présence éventuelle de ses harmoniques et non de trois fréquences précises non multiples les unes des autres. De plus la fréquence étant alors proportionnelle à la racine carrée de la tension, on ne devrait pas s'attendre à une émission à des fréquences très aiguës et constante dans le temps mais plutôt à des raies larges et dont la fréquence varie dans le temps comme la tension de diode. Enfin on s'explique mal dans ce cas le maximum d'émission pour une pression de l'ordre de $10^{-2}$ torr.

4.2 MASER SYNCHROTRON SELF CONSISTENT. - Le deuxième caractère important $d$ 'un faisceau intense d'électrons relativistes est son champ magnétique. Ce champ magnétique est de l'ordre du Tesla et azimuthal. Une étude de transfert du faisceau dans un modèle simple mais self consistent [10] montre que le faisceau a une fréquence plasma qui peut être relativement uniforme mais que le courant est essentiellement transporté par une couche mince d'électrons presque périphériques. Une étude numérique des trajectoires souvent complexes de ces électrons montre que les électrons de la couche mince porteuse du courant effectuent un mouvement de rotation accompagné d'un entraînement dans la direction du faisceau et qu'ils possèdent une grande énergie perpendiculaire $\left({ }^{1}\right)$. On peut donc penser qu'un mécanisme de maser synchrotron $[4,5,6]$ est possible, le champ magnétique étant alors produit par le faisceau luimême.

En faveur de cette théorie, on peut noter que les raies très étroites observées, indépendantes de la pression du gaz et du champ magnétique longitudinal, peuvent être très voisines des fréquences de coupure $\mathrm{du}$ guide circulaire (contenant le faisceau) pour différents modes, comme on l'observe dans le maser synchrotron classique.

De plus, une étude expérimentale sommaire, reprise avec une machine de courant plus faible, montre qu'il existe une valeur critique du courant du faisceau au-dessous de laquelle l'émission ne se produit plus. La fréquence synchrotron des électrons périphériques correspondante est alors de l'ordre des fréquences de coupure des premiers modes TE et TM du guide d'onde.

Notons également que la neutralisation en charge du faisceau nécessaire à son transport s'opère pour une pression voisine de $10^{-3}$ torr alors que la neutralisation en courant qui supprime le champ magnétique propre a lieu vers 1 torr. C'est donc bien entre ces deux limites de pression que l'émission du type maser synchrotron self consistent peut être optimale.

5. Conclusions. - Bien qu'il soit indispensable d'effectuer des mesures complémentaires pour prouver la nature de l'émission nous pouvons déjà observer les caractéristiques suivantes :

- L'émission obtenue est de très grande puissance, égalant au moins les émissions les plus intenses

(1) Lequeau D., Communication privée. 
rapportées à ce jour entre 9 et $14 \mathrm{GHz}$ (environ 1 à $2 \mathrm{GW}$ ).

- L'émission comporte trois raies très étroites à 9,11 et $14 \mathrm{GHz}$.

- Ces trois fréquences sont pratiquement indépendantes de la pression du gaz et du champ magnétique.

La charge d'espace du faisceau et son champ magnétique propres sont tous deux susceptibles de fournir des mécanismes d'émission cohérente mais les éléments dont nous disposons actuellement ne nous permettent pas de trancher sur le mécanisme responsable d'autant que d'autres mécanismes du type faisceau-plasma par exemple peuvent être envisagés.

Les résultats obtenus justifient à nos yeux une étude plus approfondie qui sera reprise avec une nouvelle ligne $2 \Omega$, actuellement en construction à l'Ecole Polytechnique, assez semblable à celle utilisée dans la présente expérience.

Bien que le processus physique ne soit pas encore élucidé, on peut noter qu'il a un bon rendement : environ $2 \%$ en puissance alors que le dispositif utilisé est beaucoup plus simple que tous les dispositifs antérieurs employés pour faire des émissions intenses en microondes.

Remerciements. - Le présent travail a été effectué avec le soutien partiel du C.E.A.-D.A.M., du C.N.R.S. - et de l'Ecole Polytechnique. Les résultats obtenus ont conduit au Brevet France no 7712987 du 29 avril 1977.

\section{Bibliographie}

[1] Nation, J. A., Appl. Phys. Lett. 17 (1970) 491.

[2] Friedman, M., Appl. Phys. Lett. 26 (1975) 366.

[3] Bekefi, G., ORZeChOWSKI, T. J., P.R.L. 37 (1976) 379.

[4] Granatstein, V. L., Herndon, M., Carmel, Y., Nation, J. A., Plasma Phys. 17 (1975) 23.

[5] Granatstein, V. L., Herndon, M., Parker, R. K., SpranGLE, P., IEEE J. Quant. Elect. QE 10 (1974) 651.

[6] Granatstein, V. L., Sprangle, P., Parker, R. K. and HernDON, M., J. Appl. Phys. 46 (1975) 2021.
[7] Alfven, H., Phys. Rev. 55 (1939) 425.

[8] Rabinovich, M. S. and Rukhadze, A. A., Sov. J. Plasma Phys. 2 (1976) 397.

Humphries, S. et al., J. Appl. Phys. 46 (1975) 187.

[9] Humphries, S. et al., Appl. Phys. Lett. 25 (1974) 20.

MAHAFFEY, R. A. et al., NRL memorandum Report, May 1977 3504.

[10] Hammer, D. A. and Rostoker, N., Phys. Fluids 13 (1970) 1831. 